\title{
Imagery ability and the acquisition and retention of movements
}

\author{
SUSAN GOSS, CRAIG HALL, and ERIC BUCKOLZ \\ University of Western Ontario, London, Ontario, Canada \\ and \\ GRAHAM FISHBURNE \\ University of Alberta, Edmonton, Alberta, Canada
}

\begin{abstract}
In this study, we examined the relationship between imagery ability, as measured by the Movement Imagery Questionnaire (MIQ), and the acquisition, retention, and reacquisition of movements. Based on their MIQ scores, 10 subjects were selected for the following imagery groups: high visual/high kinesthetic $(\mathrm{HH})$, high visuallow kinesthetic $(\mathrm{HL})$, and low visual/low kinesthetic (LL). The subjects learned four movements to a criterion level. Before each trial, subjects kinesthetically imaged the movement about to be produced. Following each acquisition trial, subjects were provided visual feedback. The acquisition phase was followed by a 2-day retention interval, a retention test consisting of three trials on each movement (no feedback provided), and a reacquisition phase. The HH group acquired the movements in the least number of trials, the LL group required the greatest number of trials, and the HL group required an intermediate number of trials. The data for the reacquisition phase showed the same trend. There was only weak evidence for a relationship between imagery ability and the retention of the movements. These findings support the position that high imagery ability facilitates the acquisition, but probably not the short term retention, of movements.
\end{abstract}

Is there an advantage to being a high imager? This question has been addressed since the early days of imagery research. One reason for the interest may be that virtually everyone seems to have the ability to generate and use images, but not to the same degree. When asked to form an image of a common object (e.g., a kite), some people find the mental task very easy and report having a vivid image, whereas others experience more difficulty and report having an image that is vague. It would seem to follow, therefore, that on a task for which imagery should be useful, high imagers would have an advantage. This has not always proven to be the case. Although positive results certainly have been reported (see Ernest, 1977, for a review), a number of studies have been unsuccessful in showing a relationship between variations in imagery ability and task performance (see J. T. E. Richardson, 1980, pp. 117-142). This is especially true in studies in which motor tasks have been employed.

Start and A. Richardson (1964) investigated the relationship between scores obtained on both the Test of Visual Imagery Control (TVIC; Gordon, 1949) and an inventory based on the Questionnaire Upon Mental Imagery (QMI; Betts, 1909) and the learning and performing of a gymnastic skill. They found no evidence that either controllability or vividness of imagery predicted

This research was supported in part by Grant No. P01 4A5 from the Natural Sciences and Research Council of Canada. Correspondence should be addressed to Craig Hall, Faculty of Physical Education, University of Western Ontario, London, Ontario, Canada N6A 3K7. performance scores on the motor task. There was some evidence, however, that vivid imagers who did not have control of their imagery performed more poorly than all other subjects. Epstein (1980) considered imagery ability in a study that examined the effects of imagery as a form of mental preparation prior to performance. Each subject was required to complete a questionnaire that involved creating four images and rating each image on clarity and difficulty; on the auditory, olfactory, tactile, and kinesthetic sensations created; and on his/her ability to concentrate on the image. When the relationship between the scores obtained on the questionnaire and the accuracy on the performance task (dart throwing) was examined, performance for males was positively correlated to auditory, tactile, and olfactory scores, whereas performance for females showed only a weak positive relationship to auditory imagery.

One recent study has reported somewhat more positive results. Ryan and Simons (1982) studied improvement following mental or physical practice in learning to balance on a stabilometer. At the completion of the learning phase, subjects in the mental imagery conditions answered a questionnaire concerning the amount and quality of any visual or kinesthetic imagery they had experienced. It was found that physical practice produced larger improvements than did mental rehearsal, and both were better than no practice. Performance of subjects who were asked to use imagery in mental rehearsal was superior to that of subjects asked not to. Also, subjects reporting strong visual images showed more improvement than those with weak 
visual images, and those reporting strong kinesthetic images were better than those with weak kinesthetic images.

Several studies have examined the relationship between imagery ability and the memory of movement attributes. Housner and Hoffman (1978) classified subjects as high visual imagers and low visual imagers, based on the subjects' scores on Marks's (1973) Vividness of Visual Imagery Questionnaire (VVIQ), and then compared their abilities to reproduce criterion end locations on a curvilinear positioning task. High imagers more accurately reproduced end locations than did low imagers, and vividness ratings of end locations correlated substantially with reproduction accuracy for high imagers, but not for low imagers. In a second study, Housner and Hoffman (1981) compared the performances of high and low imagers, categorized by their scores on the space relations section of the Differential Aptitute Test (Bennet, Seashore, \& Wesman, 1959), on the reproduction of location and distance cues from simple angular movements. Four retention conditions were included: immediate reproduction, rest, imaginal rehearsal, and imaginal distraction. High imagers more accurately reproduced movement locations than did low imagers in the immediate reproduction and imaginal rehearsal conditions, but only when scores of high imagers reporting the use of an imaginal coding strategy were compared with scores of low imagers not using such a strategy.

In contrast to the above two studies, studies by other researchers have failed to show any relationship between imagery ability and memory of movement attributes. Walsh, Russell, and Imanaka (1980) found no relationship between scores obtained on the QMI and the reproduction of location and distance information. Kakoschke and Roy (1985) investigated changes in the level of excitation of the alphamotoneuron pool (Hoffman reflex) due to imagining the production of various movements at three levels of force. Imagery ability was measured using the QMI and a questionnaire developed to determine imagery ratings for tasks involving a high component of force. The predictions that high imagers would be able to image the forces more clearly and accurately, which would allow for faster learning and more accurate recall of the forces, and that they would show significant changes in the Hoffman reflex during imaged force reproduction, were not supported. Although changes in the Hoffmann reflex during imagery were shown, these changes were not related to imagery ability as measured by the imagery questionnaires. In addition, the ratings of imagery ability were not correlated with either the ability to learn the forces or the accuracy with which they were remembered.

The studies mentioned above certainly demonstrate that researchers to date have failed to show clear-cut and consistent relationships when variations in imagery ability are used to predict motor task performance. Hall, Pongrac, and Buckolz (1985) recently suggested some possible reasons for these inconsistent findings. They contend that the instruments employed to measure imagery ability have been inappropriate. These instruments (e.g., VVIQ) are not concerned with movement, and it is likely that people classified as high or low imagers, based on their im- agery ratings of people, places, and scenes, might not differ in their abilities to image movements. In addition, kinesthetic imagery, possibly very important in imagery of movements, often has not been assessed. There also has been a failure to classify individuals jointly on both visual and kinesthetic imagery, and it may be that low visual imagery ability is compensated by high kinesthetic ability, or vice versa.

In the present study, we once again examined the relationship between imagery ability and motor skill performance but, unlike previous researchers, we employed an instrument especially designed to assess individual differences in imagery of movement. Specifically, subjects were administered the Movement Imagery Questionnaire (MIQ; Hall \& Pongrac, 1983) and then were classified into groups of high and low imagers according to both their visual and kinesthetic imagery scores. The imagery groups were then compared on their acquisitions and retention of four movement patterns. It was expected that high imagers would acquire the movements more rapidly and remember them better than would low imagers.

\section{METHOD}

\section{Subjects}

Subjects were drawn from the Sport Western Summer Program at the University of Western Ontario and the general university population. Their ages ranged from 10 to 40 years with a mean age of $22.2 \pm 5.46$, and all subjects participated on a volunteer basis. In total, 219 subjects completed the MIQ, and 30 of these subjects also participated in the acquisition and retention phases of the study.

\section{Materials and Apparatus}

The MIQ was employed for the measurement of both visual and kinesthetic imagery ability, in order to classify subjects into one of four groups. The MIQ consists of 18 items designed specifically to measure imagery of movement. Each item in the questionnaire is a separate movement, and each movement is specifically described so that every person completing the questionnaire is imaging the same movements. A variety of arm, leg, and whole body movements are incorporated in the MIQ, and all are relatively simple in order to ensure that most individuals can perform them.

Completing an item on the questionnaire requires several steps. First, the starting position for a movement is assumed. Second, the movement is produced as described. Third, the starting position is reassumed, and producing the movement is imaged (no movement is actually performed). Finally, a value is assigned from a 7-point rating scale regarding the ease/difficulty with which the movement was imaged. A low rating indicates that a movement was easy to image, and a high rating indicates that a movement was difficult to image. ${ }^{1}$

For the experimental task, four movement patterns were needed. A pilot study was conducted to help us select the four patterns. Twenty-seven movement patterns were subjected to three types of rating by 50 male and female university students. Subjects rated the ease/difficulty in labeling, visually imaging, and kinesthetically imaging the patterns using a 7-point rating scale (7 representing very easy to image or label and 1 representing very hard to image or label). Subjects were presented each movement pattern on a $180^{\circ}$ pantograph (described below). The movement patterns were presented by the experimenter who moved one end of the pantograph while the subjects passively held the other end. The patterns were presented in random order. Following the presentation of each movement pattern, subjects gave their verbal ratings. Descriptive 
statistics performed on the ratings allowed four patterns to be selected on the basis of their imagery and labeling values. The criterion for this selection was that the patterns had to have similar values (within $15 \%$ of each other on the rating scale) for a given type of rating. It was thought to be necessary to control for the assessed stimulus characteristics of the patterns, because previous research (Hall, 1980) has shown that memory for a movement pattern is related to the imagery value associated with that pattern. It was possible to choose four patterns that met this criterion. The four patterns and their corresponding imagery and labeling values are presented in Figure 1.

In the present study, these four movement patterns were performed by the subjects on the pantograph. The pantograph consisted of two metal parallelograms joined in series at a central supporting post such that movements made at one corner of the first (experimenter's) parallelogram were exactly replicated at the corresponding corner of the second (participant's) parallelogram. The pantograph was mounted in the center of a wooden platform. Attached to one corner of the experimenter's parallelogram was the cursor of a Scientific Accessories Graf Bar digitizer. The digitizer was mounted on one side of the wooden platform such that an active area $(45 \times 60 \mathrm{~cm})$ was produced in which the pantograph could be moved. When a pattern was made within this area, it was recorded by the sonic digitizer, and the digitizer converted the movement to $x$ and $y$ coordinates. The digitizer was interfaced with an NEC microcomputer via a 232 serial port. Consequently, the movements made using the pantograph were recorded and stored in the NEC computer as $x$ and $y$ coordinates for future use. Feedback concerning movements made on the pantograph was supplied to subjects via a 12 -in. monitor connected to the NEC computer and positioned directly in front of the subject at eye level. The distance between the subject and the monitor was approximately $3 \mathrm{~m}$, the pantograph being situated in this space.

\section{Procedure}

The subjects completed the MIQ individually in a quiet laboratory room relatively free from distractions. The MIQ scores were subsequently analyzed using descriptive statistics in order to select subjects who, on the basis of their visual and kinesthetic scores, fell into one of the following four imagery groups: high visual and

\section{PATTERN 1}

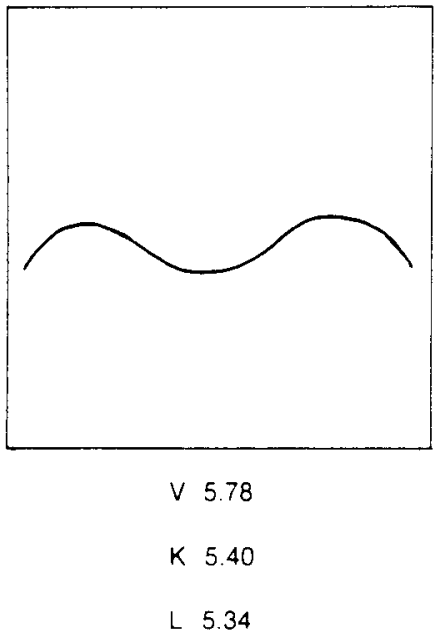

PATTERN 3

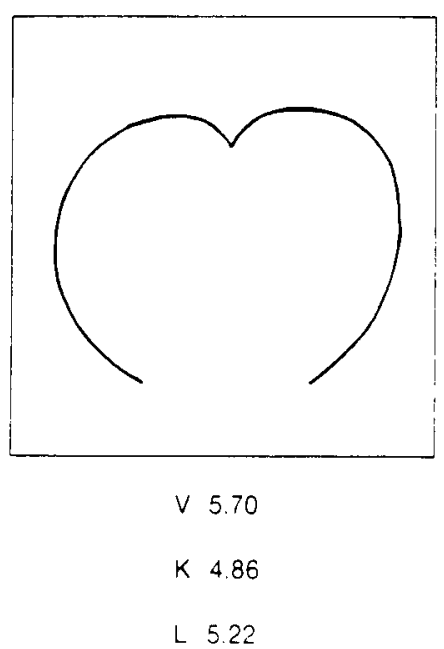

PATTERN 2

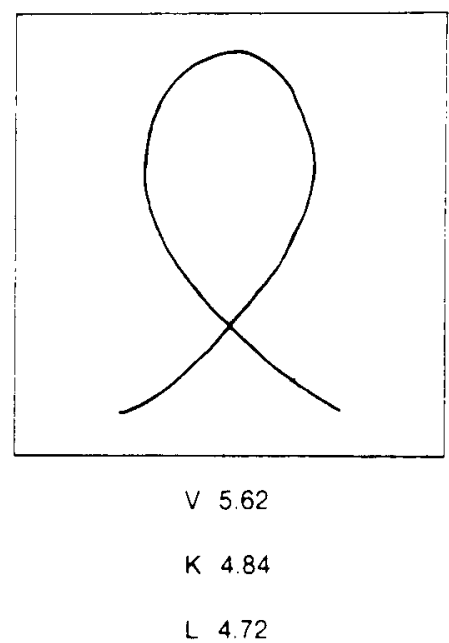

PATTERN 4

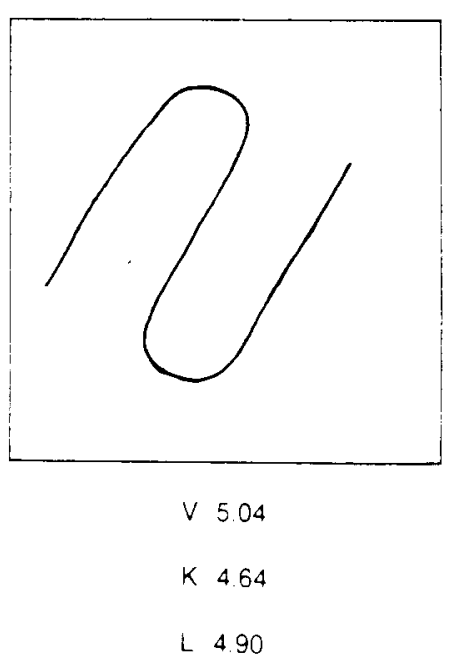

Figure 1. Mean ratings for visual imagery (V), kinesthetic imagery (K), and labeling (L) of the four movement patterns. 
high kinesthetic imagers, high visual and low kinesthetic imagers, low visual and high kinesthetic imagers, or low visual and low kinesthetic imagers. For both imagery scores, the criterion for a high imager was a score of at least $1 S D$ below the mean for the first 50 subjects completing the MIQ. Similarly, low imagers were those scoring more than $1 S D$ above the mean. ${ }^{2}$ Subjects not falling into one of these groups were eliminated from further testing.

Following assignment to groups, subjects were tested separately on the acquisition of four movement patterns. Each subject was familiarized with the pantograph, and an explanation was given regarding the task of learning to produce each of the four movements, as well as the procedure to be followed. The subject was then blindfolded, and the four criterion movement patterns were presented in a randomly assigned order by the experimenter. After the initial presentation of a movement pattern, the blindfold was removed, and the subject, regardless of group membership, was given the same imagery instructions. These kinesthetic imagery instructions required the subject to attempt to "feel" producing the correct movement pattern without actually physically doing it. Following the kinesthetic imaging of the movement pattern, the subject attempted to correctly produce that pattern on the pantograph. This attempt appeared on the monitor directly in front of the subject. Visual feedback was then presented in the form of the template (criterion pattern) that was superimposed on the monitor with the subject's attempt. The template was the correct or criterion movement pattern with a $2-\mathrm{cm}$ bandwidth around it (see Figure 2).

Following the presentation of each of the four movement patterns to the subject in the manner described above, the subject was given a series of acquisition trials using a random schedule. On each trial, the subject kinesthetically imaged the pattern to be produced, which was indicated by a verbal cue, then produced the pattern on the pantograph, and finally received feedback regarding his/her performance. A subject achieved a completely accurate (perfect) performance if his/her pattern completely matched the criterion pattern within the limits of the bandwidth. The acquisition trials continued until the subject reached the performance standard of having produced each of the four movement patterns accurately on two consecutive trials; that is, the subject had to perform a series of correct trials in which each pattern reached performance standard at least two times within this series. If two trials of a certain pattern were performed to criterion and were followed by an incorrect performance (as more patterns still had to be learned), the subject had to start over on that pattern and correctly produce the pattern two more consecutive times.

The acquisition phase was followed by a 2-day retention interval and then a retention test phase. Subjects were informed of the reten- tion phase prior to starting the acquisition phase. For the retention test phase, the subject had to produce each criterion pattern three times on the pantograph with no feedback. As in the acquisition phase, on each trial the subject was asked to form a kinesthetic image of the criterion movement pattern prior to performing the pattern. Following the third attempt at producing each pattern, the subject was provided with the same feedback as in the acquisition phase. Subjects were then asked to continue performing all four patterns in a random order with feedback provided until the performance standard was once again reached. On completion of this reacquisition test phase, subjects were debriefed and completed a short questionnaire concerning their actual use of imagery during the study.

\section{Data Analysis}

Descriptive statistics were performed on all MIQ scores. For the acquisition and the reacquisition phases of the study, one dependent variable was the number of trials required to achieve the criterion performance level. The corresponding experimental design was a 3 (imagery group) $\times 4$ (movement pattern) split-plot design. A second dependent variable was total distance error (TDE). This score measured the difference (in centimeters) between a subject's pattern and the criterion movement pattern to be produced on a given trial. ${ }^{3}$ The experimental design employed for this dependent variable was a 3 (imagery group) $\times 4$ (movement pattern) $\times 2$ (performance trial) split-plot design. The two levels of this latter variable were initial acquisition performance (first trial) and performance at criterion level (last trial). The retention phase also was examined using TDE scores in a 3 (imagery group) $\times 4$ (movement pattern) split-plot design. A second method of examining retention was by determining the number of correct attempts produced for each movement pattern and conducting a chi-square analysis on the number of subjects who correctly produced at least one movement pattern within the defined criterion limits.

\section{RESULTS}

Of the 219 subjects completing the MIQ, 22 (or 10\%) fell into the high visual/high kinesthetic (HH) imagery group, 19 (or $9 \%$ ) fell into the low visual/low kinesthetic (LL) imagery group, 11 (or 5\%) fell into the high visual/ low kinesthetic (HL) imagery group, and no subjects fell into the low visual/high kinesthetic (LH) imagery group. Therefore, the HH, LL, and HL imagery groups each contained 10 subjects selected from those having the appropri-

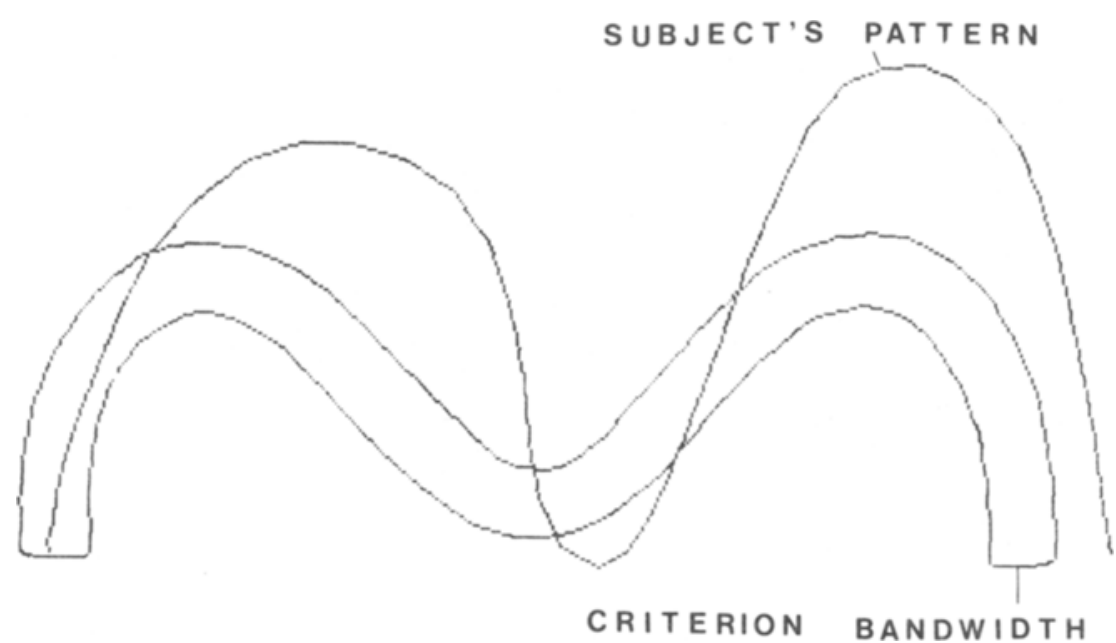

Figure 2. An example of the feedback given to subjects on each trial of the acquisition phase. 
Table 1

Means and Standard Deviations of MIQ Scores Calculated for the Subjects in the Three Imagery Groups

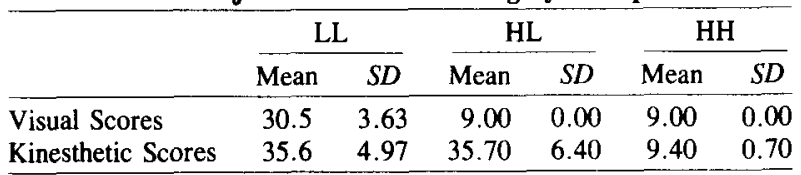

Note $-\mathrm{LL}=$ low visual/low kinesthetic group; $\mathrm{HL}=$ high visual/low kinesthetic group; $\mathrm{HH}=$ high visual/high kinesthetic group.

ate imagery scores, but no subjects fit the criterion of being low visual/high kinesthetic imagers. Means and standard deviations of the MIQ scores for each of the three imagery groups (10 subjects per group) are presented in Table 1.

Frequency distributions of the visual and kinesthetic imagery scores on the MIQ for the total sample are reported in Figures 3 and 4, respectively. The two distributions are similar in that both are positively skewed toward the high imagery scores. The majority of subjects scored as high imagers in both the visual and kinesthetic imagery sections. On the visual imagery section of the MIQ, 46 subjects (or $21 \%$ ) scored as high visual imagers, and on the kinesthetic imagery section, 44 subjects (or $21 \%$ ) scored as high kinesthetic imagers. With respect to the low scoring end of each of the distributions, 32 subjects (or $14 \%$ ) were low visual imagers, and 46 subjects (or $21 \%$ ) scored as low kinesthetic imagers. A correlation analysis of the visual scores with those of the kinesthetic scores produced a coefficient of $.63(p<.01)$. This is similar to the value reported by Hall et al. (1985) and indicates that visual and kinesthetic imagery of movement, as assessed by the MIQ, are related but separate measures.

For the acquisition phase of the study, an analysis of variance of the number of trials showed a significant group effect $[F(2,27)=14.54, p<.0005]$. Further analysis with the Tukey test indicated a significant difference $(p<.05)$ among all three imagery groups. The $\mathrm{HH}$ imagery group learned the movement patterns in the least number of trials (mean $=11.00$ ), whereas the LL imagery group required the greatest number of trials (mean $=23.73$ ). The mean number of trials for the HL imagery group was 15.40 . The main effect for movement pattern and for the group $\times$ movement pattern interaction both failed to be significant $(p>.05)$.

A similar analysis of the trial data for the reacquisition phase just failed to produce a significant group main effect $[F(2,27)=2.79, p<.08]$. The trend, however, was the same as for the acquisition phase, with a mean number of trials of 6.30 for the $\mathrm{HH}$ imagery group, 6.73 for the HL imagery group, and 9.18 for the LL imagery

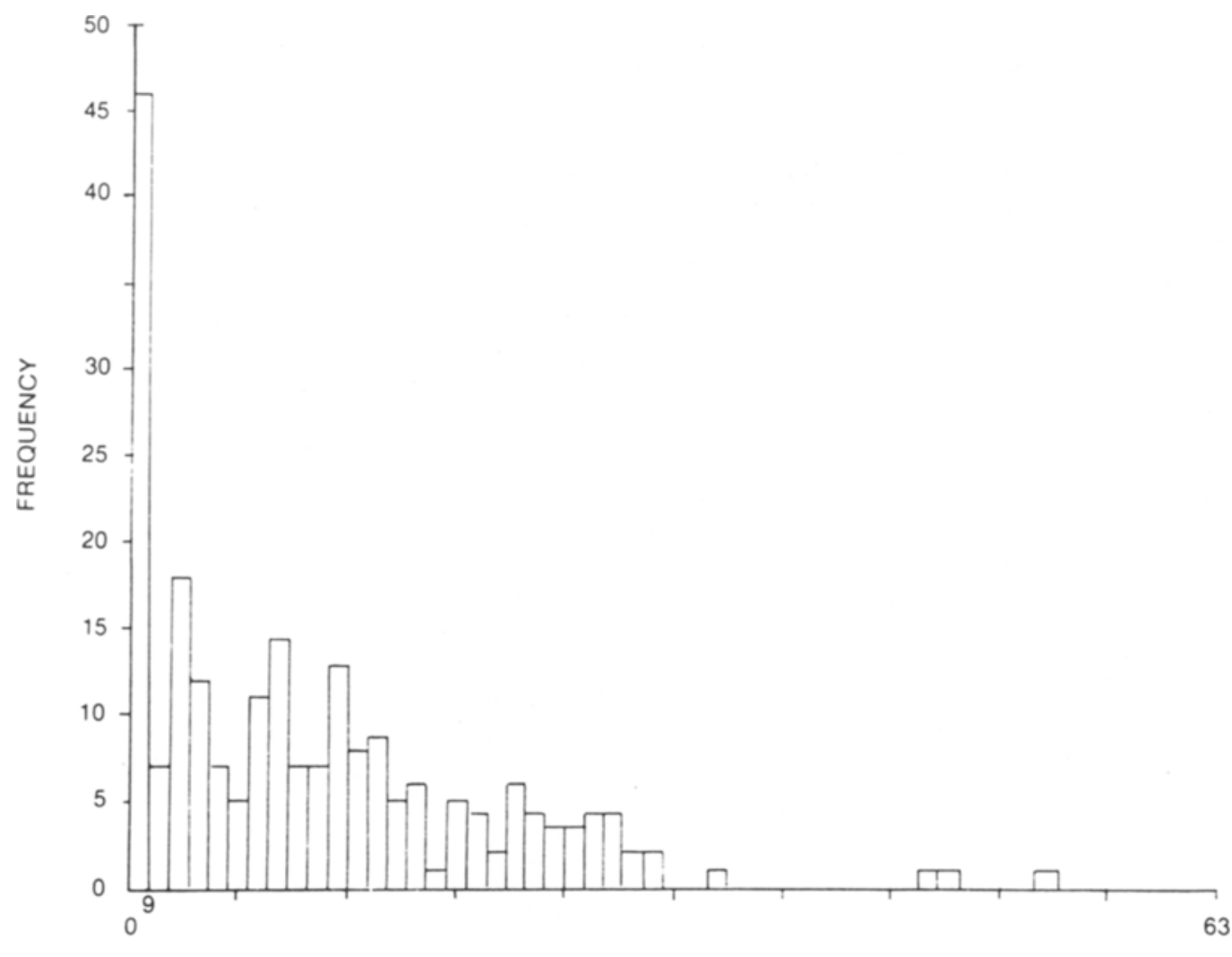

VISUAL SCORES ON THE MIQ

Figure 3. Distribution of 219 visual imagery scores on the Movement Imagery Questionnaire. 


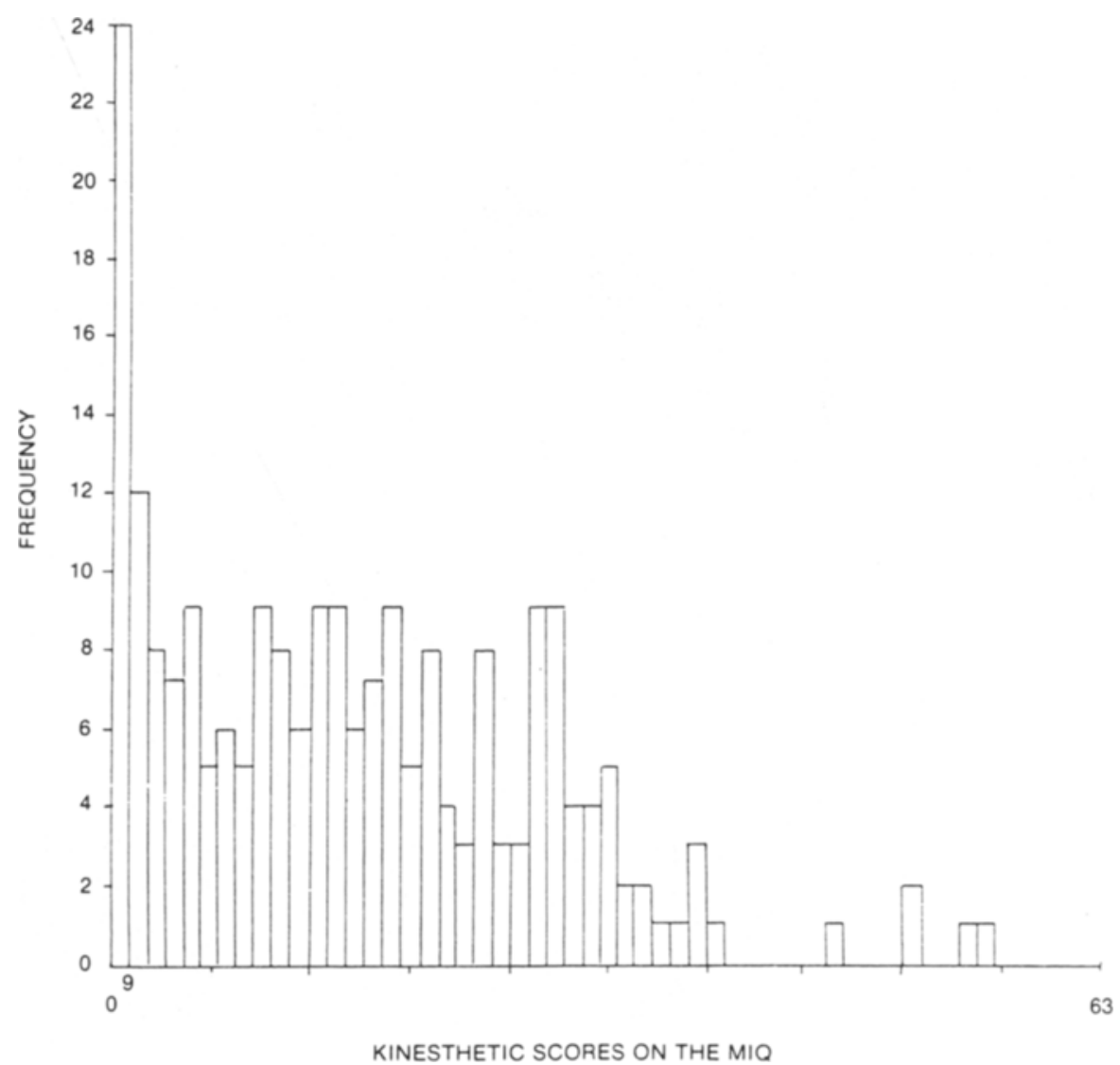

Figure 4. Distribution of 219 kinesthetic imagery scores on the Movement Imagery Questionnaire.

group. There again proved to be no significant main effect for movement pattern and no significant group $\times$ movement pattern interaction $(p>.05)$.

The analysis of the TDE scores for the acquisition phase failed to show a significant group main effect or any significant interactions involving this variable $(p>.05)$. This is important since it indicates that all the groups had similar performance levels at the start of the acquisition phase. The initial mean TDE score was $166.63 \mathrm{~cm}$ for the $\mathrm{HH}$ group, $164.05 \mathrm{~cm}$ for the HL group, and $178.85 \mathrm{~cm}$ for the LL group. The only significant main effect for the acquisition phase proved to be performance trial $[F(1,25)=207.97, p<.001]$; this was to be expected since it shows that all groups decreased their TDE scores from the start $($ mean $=169.85 \mathrm{~cm})$ to the end (mean $=55.56)$ of this phase. There also was a significant movement pattern $\times$ performance trial interaction $[F(3,75)=4.44, p<.01]$, as shown in Figure 5. Initially, not all patterns were performed within the same degree of accuracy; however, any significant differences were eliminated by the end of the acquisition phase. It should be noted that TDE did not equal zero at the termination of the acquisition phase, since subjects were not required to perform the criterion movement patterns perfectly, but just within the criterion bandwidths that were established. For the reacquisition phase, all main effects and interactions failed to reach the conventional significance level $(p>.05)$.

A point of interest in the retention phase was whether subjects could produce the movement patterns correctly (within the bandwidth limits). A chi-square analysis of the number of subjects in the three imagery groups who correctly produced at least one of the movement patterns proved to be significant $\left[\chi^{2}(2)=7.09, p<.03\right]$. Half (5) of the subjects in the HH imagery group produced patterns during retention trials within the limits designating correct retention, 2 subjects in the HL imagery group performed at this level, and no subject in the LL imagery group correctly performed any of the movement patterns. Although these results provide some evidence for a relationship between imagery ability and retention, an analysis of the TDE scores failed to reveal any significant differences either among the imagery groups or the movement patterns $(p>.05)$. This probably was because overall retention performance was quite good for each of the groups $(64.31 \mathrm{~cm}$ for the $\mathrm{HH}$ imagery group, $64.43 \mathrm{~cm}$ for the HL imagery group, and $71.82 \mathrm{~cm}$ for the LL imagery group) in comparison with their performance at the completion of the acquisition phase.

The questionnaire that subjects completed following the reacquisition phase revealed that subjects experienced little difficulty in complying with the kinesthetic imagery in- 


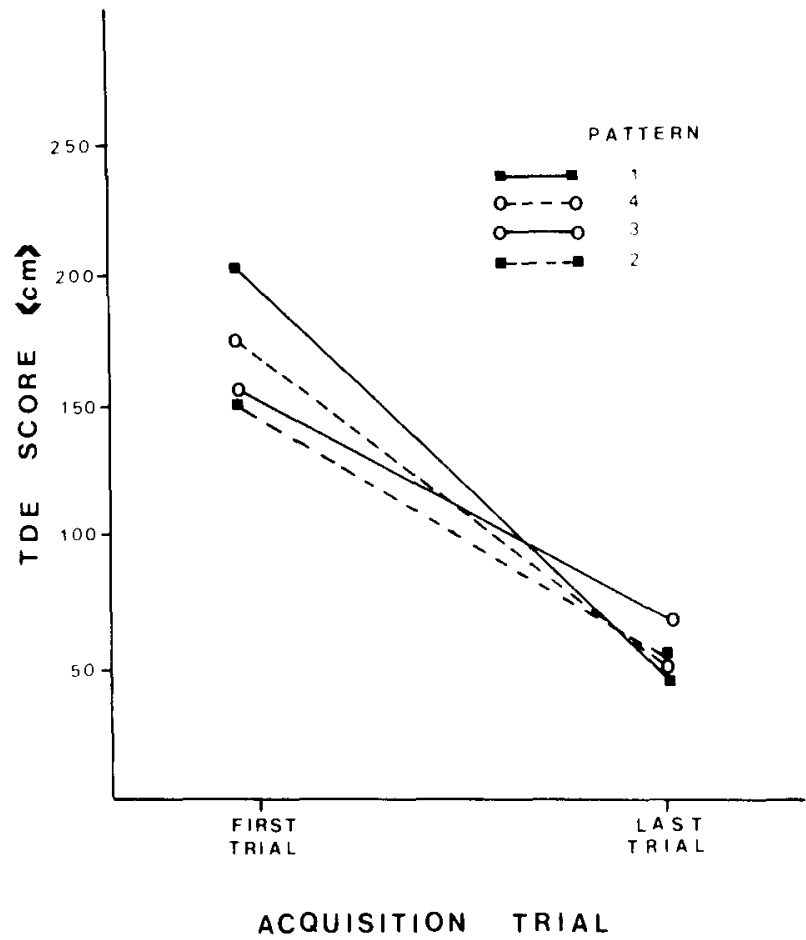

Figure 5. Mean total distance error for the four movement patterns at the beginning and end of the acquisition phase.

structions. As would be expected, those in the $\mathrm{HH}$ imagery group reported the least difficulty in kinesthetically imaging the movement patterns, whereas those in the LL imagery group had the most difficulty in doing this task. None of the subjects, however, indicated that they had substantial difficulty in following the experimental procedures or that they experienced any other problems that would suggest they be omitted from the study.

\section{DISCUSSION}

Studies by researchers who have attempted to demonstrate a relationship between individual differences in imagery ability and the learning and performance of motor tasks have yielded ambiguous results. Part of the reason for the equivocal findings lies in the problem of determining the best instrument to be employed in measuring the individual differences. The MIQ has been suggested (Hall et al., 1985) as a possible tool for measuring differences in both visual and kinesthetic imagery ability of movement. Consequently, the MIQ was employed in the present study and completed by a large number of subjects representing a wide age range. It was found that for both imagery scales, high imagers scored 9,10 , or 11 , whereas low imagers had a score of at least 26 . The mean score for visual imagery was $17.48 \pm 8.40$, and the mean score for kinesthetic imagery was $21.90 \pm 10.30$. In comparison with results from previous research employing the MIQ and conducted on athletes, the values obtained here are higher. For example, in a study recently conducted on subjects of varying levels of figure skating abil- ity (Mumford \& Hall, 1985), the means were $15.56 \pm 5.83$ for kinesthetic imagery and $15.69 \pm 5.91$ for visual imagery. This suggests that, as might be expected, trained athletes have better (more highly developed) imagery skills for movement than does the average population, at least as measured by the MIQ.

As illustrated in Figures 3 and 4, the distributions of both the visual and kinesthetic scales on the MIQ are skewed toward the high imagery scores. This may be an indication that although the ability to use imagery may fluctuate, all humans possess the potential to image and most likely employ this potential to a greater degree than is realized until forced to rate it on a questionnaire (such as the MIQ). This frequency distribution data also suggests that it is much more common to be either a $\mathrm{HH}$ imager or LL imager than to fall into one of the two combined groups, HL or LH imagers. The majority of the sample did not fall into any of these imagery groups, but were in what might be labeled the intermediate categories; scoring in the intermediate range on both imagery scales, or scoring in the intermediate range on one scale and either high or low on the other scale. A reflection of this point is found in the number of subjects falling into the four imagery groups in the present study; only $24 \%$ of the total sample tested fit into one of these groups. One quite unexpected finding was that no subjects were found to be LH imagers. Persons possessing low degrees of visual imagery ability and at the same time being highly developed in their kinesthetic imagery ability appear to be few and far between. Vision is often referred to as the "queen of the senses," and it is difficult to conceive of movement situations in which kinesthetic imagery ability would be developed independent of visual imagery ability, except for people with vision deficits. That is not to say that kinesthetic imagery could not be more highly developed than visual imagery. In Mumford and Hall's (1985) study on figure skaters, the senior skaters scored higher on kinesthetic imagery ability than on visual imagery ability. Elite athletes in sports that demand a highly developed kinesthetic sense, such as figure skating or diving, may be examples of people whose kinesthetic imagery ability does become highly developed.

The inclusion of kinesthetic imagery ability separates the present study from many previous studies seeking to show a relationship between the individual differences in imagery ability and motor performance, since these previous studies concentrated on visual imagery ability. The value of examining the kinesthetic imagery component when the task involves movement is given some support by the results of the acquisition phase. It was found that the HH imagery group learned the four movement patterns significantly faster than did the HL imagery group, who in turn learned the patterns in fewer trials than did the LL imagery group. Clearly, imagery ability is related to acquiring certain motor skills. Furthermore, high kinesthetic imagery ability seems to augment high visual imagery ability and may be beneficial to acquisition. It 
is important to realize that since visual feedback was employed in the present study, the kinesthetic imagery component was probably not fully emphasized in the HL condition. This may account for the significant, but fairly small, difference between performances of the HL and LL imagery groups. Larger differences between the performances of these two groups might be demonstrated if a procedure were employed in which feedback was supplied kinesthetically instead of visually. Nevertheless, the present results suggest that it is important to assess both types of imagery ability if a comprehensive investigation of the relationship between individual differences in imagery and motor skill acquisition is to be undertaken. The present results also support the notion that the visual and kinesthetic components of imagery are independent, but related, abilities. They are independent since if one ability level is held constant (e.g., high visual imagery), changes in the level of the other ability (e.g., high vs. low kinesthetic imagery) will markedly affect the acquisition rate of movement. They are related, however, because even though the subjects kinesthetically imaged the movements, being high in visual imagery ability still proved to be very beneficial in movement acquisition (the HL imagery group learned the patterns in fewer trials than did the LL imagery group).

The results of the analysis of the retention phase data provided only weak support for a relationship between imagery ability and the memory of movement patterns. Although more $\mathrm{HH}$ subjects than HL subjects were able to produce the movement patterns correctly (after the 2day retention interval) and none of the LL subjects could correctly produce any of the patterns, the overall accuracy (as measured by TDE) with which the patterns were remembered did not differ among the three imagery groups. These results suggest that high imagers have little advantage over low imagers in the retention of movements. It is possible that this might be true, however, only for fairly short retention periods. Retention performance, at least in terms of TDE scores, indicated relatively little forgetting of the movements by subjects in all three imagery groups over a short retention period, but following a longer retention interval, high imagers might retain a better memory of the movements. This good retention performance would account also for why no differences were evident between high and low imagers for the reacquisition phase.

The results of the present study were encouraging for a number of reasons. The MIQ was provided with additional support as an instrument proficient in classifying people according to two types of imagery ability (visual and kinesthetic). Additionally, the study supported the argument previously made by Mumford and Hall (1985) that kinesthetic imagery is an important component that must be considered when attempting to establish the relationship between motor skill performance and individual differences in imagery ability. These results are in accord with Ryan and Simon's (1982) study involving physical and mental practice effects on subjects learning to balance on a stabilometer. They found that subjects reporting strong visual images improved more than those reporting weak visual images and, similarly, subjects reporting strong kinesthetic images were better at performing the task than were those reporting weak kinesthetic images.

The reason for the success of the present study in showing a relationship between imagery ability and motor performance, where several other studies (e.g., Epstein, 1980; Start \& Richardson, 1964) have failed, may be twofold. First, the imagery test was designed specifically for imagery of movement. Previous studies may have shown positive results had they employed an instrument more sensitive in measuring visual and kinesthetic movement imagery ability. Second, the task employed in the present study emphasized the use of imagery. Subjects were specifically instructed to kinesthetically imagine producing the correct movement before each trial. The difficulty of the task allowed differences between the subjects' varying levels of imagery ability to surface. There is the possibility that in some previous studies either the use of imagery in completing the task was not sufficiently stressed, or the task was so simple it did not benefit from imagery even though imagery was used. Because imagery, including kinesthetic imagery, was emphasized throughout the present study, we believe that these results accurately reflect the strong relationship that can exist between imagery ability and the learning of a motor task.

\section{REFERENCES}

Bennet, G. K., Seashore, H. G., \& Wesman, A. G. (1959). Differential aptitude test manual (3rd ed.). New York: Psychological Corp.

BETTS, G. H. (1909). The distribution and functions of mental imagery. New York: Teachers College, Columbia University.

Epstein, M. L. (1980). The relationship of mental imagery and mental rehearsal to performance of a motor task. Journal of Sport Psychology, 2, 211-220.

ERNEST, C. H. (1977). Imagery and cognition: A critical review. Jour. nal of Mental Imagery, 2, 181-216.

Gordon, R. (1949). An investigation into some of the factors that favou the formation of stereotyped images. British Journal of Psychology 40, 156-157.

HALL, C. R. (1980). Imagery for movement. Journal of Human Move. ment Studies, 6, 252-264.

HALl, C. R., \& PongRaC, J. (1983). Movement Imagery Questionnaire London, Ontario: University of Western Ontario.

Hall, C., Pongrac, J., \& Buckolz, E. (1985). The measurement o imagery ability. Human Movement Science, 4, 107-118.

Housner, L., \& HofFman, S. J. (1978). Imagery and short-term mo tor memory. In G. C. Roberts \& K. M. Newell (Eds.), Psycholog. of motor behavior and sport (pp. 182-191). Champaign, IL: Humal Kinetic Publishers.

Housner, L., \& Hoffman, S. J. (1981). Imagery ability in recall o distance and location information. Journal of Motor Behavior, 13 207-223.

KAKOSCHKE, I., \& Roy, E. (1985). Motor imagery: Effects on the $H$ reflex. Unpublished manuscript, University of Waterloo.

Marks, D. F. (1973). Visual imagery differences in the recall of pic tures. British Journal of Psychology, 64, 17-24.

Mumford, P. B., \& HALl, C. R. (1985). The effects of internal an external imagery on performing figures in figure skating. Canadia. Journal of Applied Sport Sciences, 10, 171-177. 
RichARDSON, J. T. E. (1980). Mental imagery and human memory. New York: St. Martin's Press.

Ryan, E. D., \& Simons, J. (1982). Efficacy of mental imagery in enhancing mental rehearsal of motor skills. Joumal of Sport Psychology, 4, 41-51.

StART, K. B., \& RichaRdson, A. (1964). Imagery and mental practice. British Journal of Educational Psychology, 34, 280-284.

Walsh, D., Russell, D., \& Imanaka, K. (1980). Memory for location and distance information and imagery ability. Acta Psycholog$i c a, 44,117-130$.

\section{NOTES}

1. The development of the MIQ and the appropriate statistical analyses are reported in Hall et al. (1985). The instrument has an equal number of items in the two subscales (visual and kinesthetic), and these two subscales have moderate, positive correlations $(.20$ to .30$)$ with other instruments employed to assess imagery ability (e.g., QMI, VVIQ). The MIQ is available from the second author on request and includes a set of instructions and scoring information.

2. Note that a low score on the MIQ indicates high imagery ability and that a high score indicates low imagery ability. The MIQ was designed in this manner to correspond with several other instruments used to measure imagery ability.

3. TDE was calculated for each movement produced by a subject using the Equation,

$$
\mathrm{TDE}=\sum_{1}^{n} \sqrt{\left(x_{i}-x_{j}\right)^{2}+\left(y_{i}-y_{j}\right)^{2}},
$$

where $x_{i}$ and $y_{i}$ are points on the criterion pattern, $x_{j}$ and $y_{j}$ are corresponding points on the subject's pattern, and $n$ is the number of points sampled on each pattern. For a given calculation, $x_{i}$ and $y_{i}$ were determined by the computer and based on the number of sample points comprising the criterion pattern, while $x_{j}$ and $y_{j}$ were selected by the experimenter. The experimenter was blind to group membership and experimental trial (acquisition or retention) when making all calculations.

(Manuscript received February 10, 1986; revision accepted for publication April 15, 1986.) 